\title{
Religion et piété sceptiques selon Sextus Empiricus
}

\author{
Stéphane Marchand
}

Parce que le scepticisme est une philosophie sans thèse, il peut recevoir une pluralité d'interprétations parfois contradictoires. À propos du scepticisme, un malentendu est toujours possible: aujourd'hui autant des créationnistes que des évolutionnistes, des athées que des philosophes chrétiens utilisent le scepticisme pour des buts diamétralement opposés. À ce titre, poser la question de la relation entre scepticisme et religion est une bonne occasion pour lever, autant que faire se peut, certaines indéterminations propres au corpus sceptique.

En ce qui concerne Sextus Empiricus, trois textes abordent la question de la religion et de la piété sceptiques. Dans les Esquisses Pyrrhoniennes (PH) I 23-24, Sextus explique que le sceptique n'est pas inactif contrairement à ce que prétendent les dogmatiques, qu'il agit en suivant les phénomènes c'est-à-dire qu'il vit selon « les règles de la vie quotidienne » parmi lesquelles celle « de suivre la tradition des lois et des coutumes qui fait que nous considérons la piété, dans la vie quotidienne, comme bonne et l'impiété comme mauvaise » ${ }^{1}$. Cette position se retrouve en $P H$ III 2 en préliminaire de l'enquête physique sur les dieux : «en suivant sans soutenir d'opinions les règles de la vie quotidienne nous disons qu'il existe des dieux, nous révérons les dieux et nous affirmons qu'ils exercent une providence... »; elle est répétée, enfin, dans le Contre les physiciens ( $A M$ IX), 49: "suivant les coutumes traditionnelles et les lois, il [sc. le sceptique] dit que les dieux existent et il s'applique à les servir et les respecter $»^{2}$.

Le propos de cet article est d'interroger cette position en deux temps :

1. Quelle est l'intention de Sextus quand il parle de la religion et de la piété sceptiques ? Comment caractériser la position personnelle du sceptique sur cette question ?

2. Une fois formulée une hypothèse sur cette intention, reste à tester la validité et la cohérence de cette position en relation avec le concept de piété. La piété sceptique estelle possible?

\section{La place de la religion dans le néo-pyrrhonisme}

Pourquoi sonder l'intention réelle du sceptique, comme si ce qu'il disait ne suffisait pas pour connaître sa position? Cette question se répète fréquemment pour l'interprétation du

${ }^{1}$ J'utilise la traduction de P. Pellegrin (Pellegrin 1997) pour PH, et je traduis le Contre les Physiciens (AM IX).

${ }^{2}$ Voir aussi $P H$ III, 219 sq., qui expose les désaccords dogmatiques sur «la piété et le service des dieux » mais n'évoque pas la question de la piété sceptique. Sur ces textes, voir l'article d'E. Spinelli dans le présent volume. 
scepticisme du fait de l'aspect défensif et critique des textes sceptiques. Dans ses œuvres, Sextus favorise l'exposé spécial du scepticisme, c'est-à-dire la critique du dogmatisme. De ce fait, l'interprétation de la position sceptique est problématique : il y a toujours un espace pour le jeu interprétatif, du fait de la prudence de Sextus, mais aussi de la structure même du

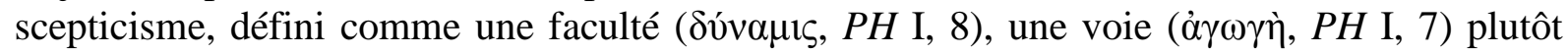

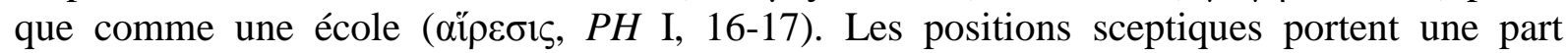
d'indétermination qui est cohérente avec la structure non thétique de cette philosophie. La question de la piété ne fait pas exception ici : Sextus ne produit aucune théorie sur la piété sceptique ; il ne saurait y avoir de $\pi \varepsilon \rho i ̀ ~ \varepsilon v ̉ \sigma \varepsilon \beta \varepsilon i ́ \alpha \varsigma$ sceptique, ni de définition précise de la piété et de la religion sceptiques.

\section{Un problème de théologie ou de philosophie de l'action?}

En $P H$ I 23-24, la question de la piété sceptique est introduite au sein d'un argument concessif permettant d'expliquer comment, sans avoir d'opinion, le sceptique peut agir, en

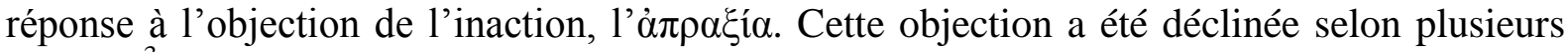
versions $^{3}$. Une version radicale consiste à montrer que le sceptique n'est pas capable de vivre, ni d'effectuer les actions minimales nécessaires à la vie humaine : aller quelque part, boire, manger, sentir, penser,... sont des actions qui requièrent, selon cette objection, d'avoir des

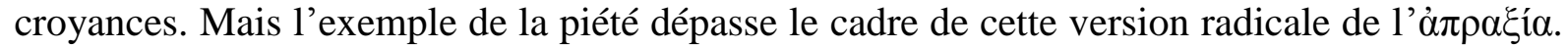
Contre cette dernière, les deux premières règles (la conduite de la nature et la nécessité des affects) suffiraient. Les deux dernières règles (tradition des lois et des coutumes, apprentissage des arts) montrent bien plutôt comment le sceptique peut vivre une vie d'homme normal dans une cité, avec un métier, etc. Pour cette raison, elles répondent probablement à une autre objection qui dénonce une contradiction entre les principes sceptiques et la vie sociale, laquelle supposerait l'acceptation de certaines règles, certaines normes sociales ou techniques.

La réponse de Sextus montre donc que l'action sceptique n'est pas réductible à un pur mécanisme pathologique, et permet des réactions plus élaborées que des actions réflexes, ou de pures répétitions. Le sceptique est capable d'agir dans un environnement complexe, dans un monde où il y a des croyances, des cultes, des valeurs qui dictent le comportement de la majorité des hommes. Selon Sextus, le sceptique est capable de s'insérer dans le monde sans pour autant partager ces croyances dogmatiques. À la différence du dogmatique, il ne suivra pas les normes sociales parce qu'il considère qu'elles sont vraies ou justes, mais parce qu'elles reposent sur un usage et une tradition. Dans la troisième règle de l'action « selon la

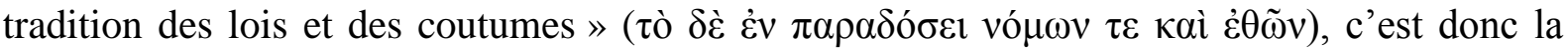
$\pi \alpha \rho \alpha ́ \delta o \sigma ı \varsigma$ qui importe, le fait que ces normes se soient transmises dans le temps, et constituent une tradition au sens propre dont la légitimité repose sur l'usage du passé ${ }^{4}$.

Qu'en est-il de la piété ? En $P H$ I, 24, la piété est simplement un exemple de cette capacité du sceptique à agir en suivant «la tradition des lois et des coutumes ». Dans ce passage, elle n'a pas d'autre valeur, et aurait pu être remplacée par un autre exemple, comme celui du tyran du Contre les moralistes (AM XI, 162 sq.). Dans les deux cas, il s'agit de montrer comment le sceptique peut accomplir des actions qui sont traditionnellement pensées à partir d'une motivation dogmatique fondée sur une croyance. La solution de Sextus consiste

\footnotetext{
${ }^{3}$ Vogt 2010.

${ }^{4}$ Corti 2009, 82.
} 
à montrer que le sceptique peut se régler sur autre chose pour agir, en se réglant sur les phénomènes et les attitudes des autres hommes à partir de l'observation empirique 5 .

La spécificité de la piété apparaît néanmoins lorsque ce texte est mis en regard avec ceux du troisième livre des Esquisses Pyrrhoniennes et du second livre du Contre les physiciens, qui servent tous deux de préliminaire aux développements physiques sur les dieux. Il ne s'agit plus alors de donner un exemple, mais de prévenir une objection particulière liée à la question des dieux et de leur existence : avant de parcourir l'ensemble des arguments pour et contre l'existence des dieux qui aboutiront à la suspension du jugement, Sextus précise que cette suspension n'a aucune incidence sur la piété sceptique. La piété constitue donc un problème isolable pour le scepticisme, qui demandera une réponse circonstanciée.

On trouve chez Aristoclès, par exemple, une accusation générale d'immoralisme sous laquelle pourrait être logée celle d'impiété :

Il faut considérer ceci : quel citoyen, quel juge, quel conseiller, quel ami, ou finalement quel homme serait un pareil individu ? Devant quels forfaits reculerait-il, lui qui ne regarde rien comme vraiment mal ou honteux, juste ou injuste ? Car qu'on n'aille pas dire que ces gens-là craignent les lois et les châtiments, avec l'insensibilité et l'ataraxie dont ils se targuent ${ }^{6}$.

Cette critique ne porte pas spécifiquement sur la religion ou la piété sceptique, mais plus généralement sur l'immoralisme pyrrhonien censé découler de l'apathie et de l'ataraxie érigées comme valeurs sceptiques. Même si Aristoclès critique une forme plus radicale de scepticisme que celui prôné par Sextus Empiricus, on peut aisément imaginer qu'une critique de ce type pourrait porter contre Sextus Empiricus quand bien même ce dernier adoucit l'apathie en métriopathie. En ce qui concerne la piété, l'objection consisterait à montrer que suspendre son jugement sur l'existence des dieux revient, d'un point de vue éthique, à refuser leur existence, puisque selon certains dogmatiques «si les dieux n'existent pas, la piété n'existe pas ${ }^{7}$. Il semble donc assez naturel de penser que la suspension du jugement sur l'existence des dieux amène à ne pas respecter les dieux, du moins à oublier de les respecter.

Pour cette raison Sextus doit prévenir l'objection qui rabat la position sceptique sur celle des philosophes athées comme ceux décrits en $A M$ IX 51-58: Évhémère, Prodicos, Diagoras, Critias, Théodore, Protagoras et Épicure ${ }^{8}$. La réponse de Sextus va montrer la différence entre le point de vue pratique et le point de vue théologique : ce n'est pas parce que le sceptique suspend son jugement sur l'existence des dieux (de fait Sextus dit que «pas davantage les dieux existent qu'ils n'existent pas»en $A M$ IX, 50 et 59) qu'il est nécessairement impie.

Par conséquent, il est possible de poser, d'abord qu'il n'y a pas de théorie explicite de la piété sceptique; ensuite, que dans les passages qui abordent la question de la piété sceptique, Sextus ne se contente pas de répondre à l'objection de l'inaction, mais répond aussi à l'accusation d'impiété ; enfin que la réponse de Sextus repose sur une distinction entre approche théologique et approche pratique de la piété.

${ }^{5}$ Cf. Spinelli 2008, 30 sq.

${ }^{6}$ Aristoclès de Messine $a p$. Eusèbe de Césarée, PE XIV, 18, 18 (trad. des Places, Cerf, 1987) ; voir aussi à propos de Pyrrhon, Aristoclès, ap. Eusèbe, Praeparatio Euangelica, XIV, 18, 27 (= Decleva Caizzi T. 23) : «il parla mal de tous, des dieux comme des hommes ».

${ }^{7} A M$ IX, 123.

${ }^{8}$ Dans le De Pietate, Philodème décrit comme des athées « ceux qui disent qu'il est impossible de savoir s'il existe des dieux », de la même façon que «ceux qui disent ouvertement que les dieux n'existent pas » et « ceux qui semblent clairement refuser leur existence » (cf. P. Hercul. 1428 col. XV. 1-8 in Henrichs 1974, voir aussi Obbink 1989, 189-190). 


\section{Trois relations possibles entre scepticisme et religion}

En quoi la réponse sceptique est-elle indéterminée ? Dans un contexte dialectique, il est possible de dégager plusieurs interprétations de cette réponse qui dessinent trois relations possibles entre scepticisme et religion.

D'abord l'interprétation « fidéiste »; cette dernière affirme que non seulement Sextus dit ce qu'il pense lorsqu'il expose la piété sceptique, mais qu'il produit une compréhension originale de ce que devrait être la piété. Vient ensuite l'interprétation « libertine » : la position de Sextus correspondrait à une «stratégie d'écriture» au sens de Léo Strauss. Le sceptique produirait cette réponse pour se mettre à l'abri d'une menace politique (comme celle qui, selon la légende, frappa Protagoras ${ }^{9}$ ). Selon cette interprétation, plutôt que de reconnaitre son athéisme, Sextus donnerait une réponse de pure convenance. Enfin, l'interprétation «indifférentiste »: les textes sur la piété sceptique n'expriment ni une compréhension de la spécificité de dieu comme objet de connaissance, ni une stratégie de dissimulation d'un athéisme. Selon cette interprétation, la position de Sextus ne saurait donc être ramenée à ces positions modernes de compréhension de la piété et de la religion sceptiques.

Quand bien même personne ne défendrait explicitement, dans la littérature critique, les deux premières interprétations, il est intéressant de les développer, ne serait-ce que pour expliciter, par différence, la position de Sextus Empiricus.

La lecture fidéiste (i) est directement liée au développement du christianisme, et plus généralement du monothéisme ${ }^{10}$. Cette interprétation trouve dans le texte de Sextus Empiricus une compréhension authentique de la piété. Sextus, en quelque sorte, anticiperait Pascal : la piété ne saurait commencer par une compréhension de Dieu, elle est d'abord portée par des gestes. Corréler l'attitude du croyant à une connaissance ou à une certitude théorétique concernant l'essence de Dieu, ou même son existence, est une façon de ne pas la comprendre. Selon cette interprétation, la piété ne dépend pas de la croyance (épistémique), au contraire c'est la croyance (religieuse) qui dépend de la piété. En distinguant la question de la croyance épistémique et de celle de la piété, Sextus préparerait le terrain pour la compréhension de la spécificité de la croyance religieuse.

Pour puissante que soit cette interprétation, il n'est pas vraisemblable que ce soit le sens du texte de Sextus. Le statut concessif de la réponse de Sextus suffit pour reconnaître que la piété n'est pas un objet privilégié pour le scepticisme néo-pyrrhonien. Sextus ne parle de la piété que parce qu'il y est contraint par une situation argumentative particulière ; cette réponse n'exprime pas le cœur de la position sceptique, ni une compréhension de la nature éminente de Dieu ou des dieux. Certes les dieux sont des objets fondamentaux, ce qui explique que l'attaque sceptique commence par eux pour que tout l'édifice s'écroule ${ }^{11}$. Mais ces objets n'appellent pas pour Sextus un mode de compréhension particulier comme le suppose l'approche fidéiste. De ce point de vue, les dieux sont des objets physiques comme les autres, au même titre que le temps, la cause, etc. que Sextus traite avec la même méthode dans les pages qu'il consacre à la physique ${ }^{12}$. Que le fidéisme trouve dans le scepticisme de

${ }^{9}$ AM IX, 55 ; sur le procès pour impiété de Protagoras et sa fuite d'Athènes, cf. Protagoras T. 1-3, édités par M. Bonazzi in Pradeau (dir.) 2009 et les p. 46-47 qui rappellent le caractère hypothétique de ces événements. Il est intéressant de noter que ce type d'interprétation a été fait pour l'épicurisme : Posidonius accuse Épicure de participer aux cultes traditionnels uniquement pour éviter une persécution politique (cf. e.g. Cicéron, De natura deorum I, 123 et les références indiquées par Obbink 1992, 185-186).

10 Il y a, en effet, un usage apologétique des arguments sceptiques chez Philon d'Alexandrie, cf. Bréhier 1907, 209-210 ; et la contribution de C. Lévy, supra, p. 000.

${ }^{11}$ Cf. Bett 2013, 159-160 pour l'intérêt de Sextus pour les « foundational questions ».

12 Cf. Sihvola 2006, 89. 
Sextus un énoncé pertinent pour penser la piété et in fine la foi n'est qu'un exemple de plus de la fécondité herméneutique de l'usage du scepticisme, indépendamment de la vraisemblance historique de cette lecture ${ }^{13}$.

Contrairement à l'interprétation précédente, la lecture « libertine » (ii) peut trouver en $A M$ IX, 49 un semblant d'appui textuel. Sextus dit, en effet, que le sceptique sera " plus en

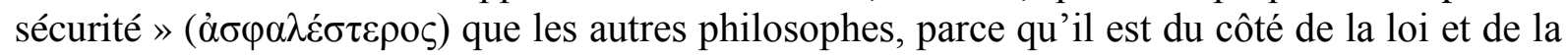
coutume. On pourrait donc interpréter cette attitude comme étant commandée par la volonté de se mettre en sécurité, de donner des gages à la pression sociale qui commande d'être pieux.

L'argument est en réalité assez elliptique ${ }^{14}$ : entre l'annonce de l'enquête sur la question de l'existence des dieux et l'énoncé selon lequel le sceptique est plus en sécurité, il y a un saut, et savoir à quoi renvoie cette sécurité n'est pas évident. Chez Sextus, l'adjectif $\grave{\alpha} \sigma \varphi \alpha \lambda \eta ́ s$ désigne d'ordinaire la sécurité épistémique, l'absence d'erreur ${ }^{15}$, et une compréhension possible pourrait être de penser que Sextus renvoie ici comme souvent à l'idée que le sceptique est en sécurité parce qu'il ne se trompe pas. Mais une compréhension politique de l'adjectif, plus cohérente avec le contexte, est possible en partant du sens du terme chez Épicure ${ }^{16}$. Ce dernier distingue, en effet, deux types de sécurité ${ }^{17}$ : celle qui est assurée par «la puissance solidement établie et l'aisance matérielle ${ }^{18}$ et qui reste vaine si elle ne s'accompagne pas d'une libération des peurs liées aux «choses d'en haut» (Maxime Capitale 13). La véritable sécurité, ou comme le dit Épicure «la plus pure des sécurités -

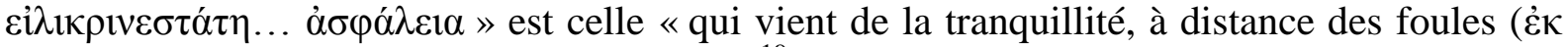

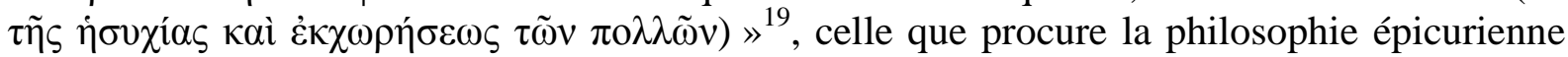
en rectifiant entre autres les conceptions erronées que les hommes se font des dieux et de leur pouvoir sur leur destinée. En contexte épicurien, cette sécurité ne se limite donc pas à une sécurité épistémique, elle suppose aussi une certaine relation vis-à-vis de la société qui fait que l'épicurien, sans être complètement coupé de la société, est dans sa communauté d'amis à l'abri de la foule et de ses passions ${ }^{20}$. Concernant l'affirmation selon laquelle le sceptique est «plus en sécurité », il me semble plus intéressant de considérer que Sextus pense ici à ce sens plus particulier de la sécurité qui implique effectivement une réflexion sur l'inclusion sociale du philosophe dans une société composé d'hommes qui ont des croyances.

$\mathrm{Si}$ on prend en considération cet aspect «politique » de la question religieuse, on constate en effet que la position épicurienne a été interprétée comme une forme dissimulée d'athéisme, comme en témoigne d'ailleurs Sextus :

Et Épicure, selon certains, admet les dieux pour le peuple, mais il ne les admet en rien pour la nature des choses ${ }^{21}$.

${ }^{13}$ Sur la relation entre le scepticisme et le fidéisme, cf. Popkin 1995 ; Penelhum 1983 ; pour une critique de la réduction du scepticisme moderne au fidéisme par Popkin, cf. Giocanti 1998.

${ }^{14}$ Pour une recension des interprétations possibles de cet argument, cf. Bett à paraître.

15 Cf. e.g. AM VII, 151 ; VIII, 300.

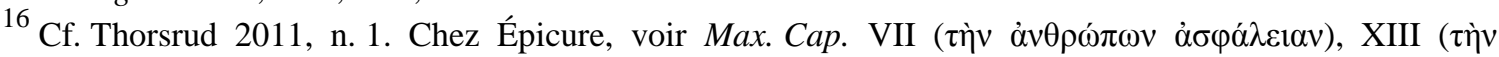

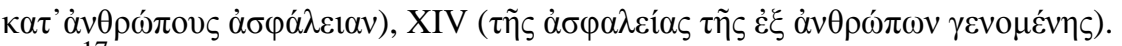

${ }^{17}$ Cf. Roskam 2007, 38-39.

${ }^{18}$ Trad. Morel. Dans ce sens, cf. l'emploi du terme par Plutarque dans le Contre Colotès 1124D.

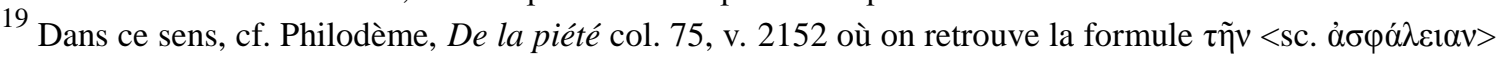
$\dot{\varepsilon} \alpha v \tau \tilde{\omega} v \pi \alpha \rho \dot{\alpha} \tau \tilde{\omega} v \pi \mathrm{o} \lambda \lambda \tilde{\omega} v$.

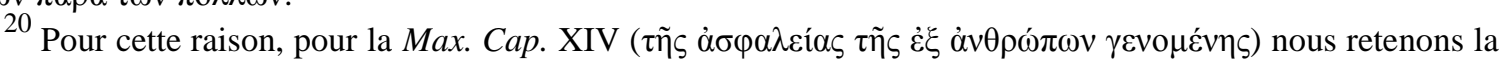
compréhension « la sécurité parmi les hommes » (cf. Balaudé 1994 et Morel 2011) et non « la sécurité contre les hommes ». Sur l'articulation entre la communauté civique et la communauté formée par l'amitié épicurienne, Morel 2007.

${ }^{21}$ AM IX, 58. Voir aussi Cicéron, De natura deorum I, 85 et III, 1. Sur cette question, voir Obbink 1989. 
En refusant l'action providentielle des dieux, les épicuriens ont donc pu être considérés comme des athées ${ }^{22}$. L'argument de Sextus sur la sécurité pourrait donc être le suivant: même si l'interprétation de l'épicurisme comme un athéisme est un contresens, le fait qu'on ait pu le comprendre comme un athéisme met le philosophe épicurien en péril, contrairement à la sécurité qu'il est censé apporter, il est exposé à la vindicte de la foule par son anti-conformisme ${ }^{23}$.

La mention de la sécurité en $A M$ IX, 49 ne correspond donc pas à une stratégie d'écriture. Il s'agit plutôt de répondre à l'objection d'impiété en montrant que le Sceptique sera plus en sécurité que les philosophes qui prétendaient offrir la plus grande sécurité, les épicuriens. D'un point de vue sceptique, l'erreur des Épicuriens est d'être allés à l'encontre des traditions. Au contraire, le philosophe sceptique, dans ses actions du moins, suit l'usage commun et ne déroge pas aux pratiques sociales de sa communauté ; il est plus en sécurité que l'épicurien car l'غ̇ $\pi \circ \chi \eta ́$ sur la question théorique de l'existence des dieux n'a aucune incidence sur son comportement religieux ${ }^{24}$.

S'agit-il d'une dissimulation ou d'un double jeu ? Pas nécessairement ; rien dans les textes ne permet d'attester d'une persécution ou d'une censure du scepticisme sur ce sujet, tout au plus pourrait-on parler d'une pression argumentative à laquelle le sceptique répond ${ }^{25}$. Ensuite, contrairement à ce que suppose ce type d'interprétation, tout est clair dans la position prise par le sceptique qui ne dissimule pas qu'il obéit aux lois et aux coutumes non pas parce qu'il croirait en leur valeur, mais tout simplement parce qu'elles se sont imposées comme lois et coutumes. La mesure de l'obéissance du sceptique n'est jamais tue, jamais dissimulée.

Il faut donc se résoudre à prendre Sextus au pied de la lettre sans lui prêter des intentions qu'il n'a pas, sans chercher à sonder ses intentions cachées, et se demander ce que signifie cette position indifférentiste vis-à-vis de la religion.

La position indifférentiste (iii) de Sextus n'est pas l'expression d'un mensonge ou d'une stratégie de dissimulation mais d'une indifférence au sens où la question de la religion n'affecte pas le cœur de la position; elle ne lui est pas essentielle. Si la piété sceptique ne tient que par les lois et les coutumes, cela signifie qu'elle est strictement conditionnée à un ensemble de conditions sociales et culturelles qui sont propres à Sextus, mais qui sont secondaires par rapport à la logique de la position sceptique ${ }^{26}$. Dans la mesure où Sextus écrit

${ }^{22}$ En $P H$ III, 10 Sextus relate par exemple l'argument selon lequel « s'il [sc. le dieu] ne veut ni ne le peut [sc. exercer sa providence], c'est qu'il est méchant et faible, ce qui est paroles impies sur le dieu ». Sur cet argument, Knuuttila, Sihvola 2000, 132 sq. et Spinelli 2916 à paraître, 4 e partie.

${ }^{23}$ On pourrait ici m'objecter que le sceptique est menacé par la même réduction à l'athéisme ; mais précisément, la réponse néo-pyrrhonienne de Sextus Empiricus constitue une réponse claire à ce type d'attaque pour défaire le lien entre scepticisme et athéisme.

${ }^{24} \mathrm{La}$ position des Épicuriens sur cette question est cependant bien plus nuancée que celle décrite ici et comporte plus de points communs que Sextus ne le reconnaît. En effet, à côté d'une réforme de la piété populaire (que l'on trouve dans les vers célèbres du livre V du DNR de Lucrèce, 1198-1203), il y a aussi une reconnaissance épicurienne du culte et des usages traditionnels comme le souligne Festugière 1997, 86-92. Voir notamment le fgt Us. 387 (apud Philodème, De la piété) : «quant à nous sacrifions pieusement et avec décence aux jours convenables, et accomplissons tous les autres actes de culte conformément aux usages ( $\kappa[\dot{i} \tau] \tilde{\alpha} \lambda \lambda \alpha$

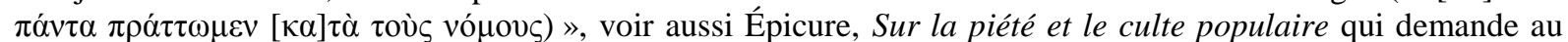
sage «d'honorer les rites religieux qui sont les tiens [sc. en tant que philosophe], en profitant des plaisirs de la

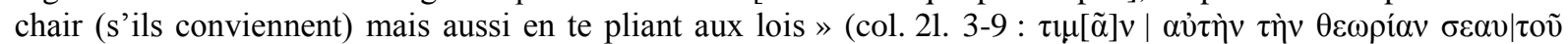

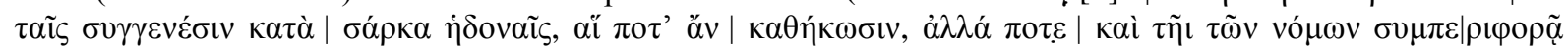
$\chi \rho \omega \mu \varepsilon ́$ vov бoṽ ; je traduis le texte donné par Obbink [1992], voir aussi Obbink 1984) ; pour une compréhension légèrement différente de ce passage, voir cependant Delattre, Pigeaud 2010, 119-120.

${ }^{25}$ Bett (à paraître), dans son analyse d'AM IX 13-194, montre que les dieux ne sont pas des objets comme les autres pour Sextus et que, pour cette raison, ce dernier insiste clairement sur la suspension du jugement pour qu'on ne croie pas qu'il refuse l'existence des dieux.

${ }^{26}$ Pour un rapprochement avec la position académicienne, cf. Knuuttila, Sihvola 2000, 137-138. 
et vit dans une société où il faut se conduire pieusement pour y tenir un rôle, avoir une existence sociale, travailler, etc., il faut adopter cette attitude pour vivre selon le modèle de la vie quotidienne. La formulation de $P H$ I, 24 est d'ailleurs intéressante à cet égard; Sextus ne décrit pas ce qu'est la piété, mais la proclame comme une valeur: «la piété est bonne, l'impiété mauvaise », tout en rappelant que cette valeur n'est adoptée que d'un point de vue phénoménal et relatif, c'est-à-dire relativement à ce qui apparaît dans la vie quotidienne, sans lui attribuer de valeur objective.

Une fois établi le cadre de cette interprétation, il faut essayer de sonder la consistance de cette approche originale de la piété, déliée de toute croyance.

\section{Qu'est-ce que la piété sceptique?}

L'enchaînement argumentatif de $P H$ I 23-24 invite à une approche pragmatique de la piété ; du point de vue du scepticisme, le problème de la piété est celui d'une action sans croyance. En montrant dans quelle mesure nous pouvons avoir un comportement pieux sans avoir de croyance, Sextus invite à penser à nouveau l'idée d'une « vie sans croyance ».

\section{Les gestes de la piété}

En quoi consiste cette compréhension pragmatique et empiriste de l'action ? De la même manière que le sceptique agit, et même parle, en suivant la voie toute tracée des hommes qu'il observe dans la vie quotidienne, pour la piété, il s'agira de dire que le sceptique participe à la religion de sa Cité ou de sa communauté en imitant ses concitoyens. Les raisons dogmatiques qui poussent ses congénères à agir importent peu au sceptique; il lui suffit de suivre la norme telle qu'elle est définie par la communauté à laquelle il appartient, en laissant reposer la charge dogmatique de la croyance sur les autres.

Il ne s'agit donc pas d'une adhésion aux valeurs de la religion, mais d'une imitation empirique qui ne relève en rien de la conviction religieuse, mais d'un acte social. Cette solution semble, de prime abord, assez naturelle dans la mesure où Sextus parle ici de la piété, c'est-à-dire avant tout d'un comportement religieux, et pas de théologie. De ce point de vue, il n'y a donc pas de différence entre l'action politique, l'apprentissage d'une technique (qui constituent d'autres exemples d'actions possibles sans opinion selon Sextus), et la piété religieuse. Cette précision doit être prise en compte dans la discussion récente sur le degré et la force de la croyance en contexte polythéiste : selon J. Annas, ce contexte impliquerait un niveau de croyance beaucoup plus faible que dans le monothéisme et expliquerait que Sextus conçoive un sceptique religieux sans contradiction. Dans la mesure où une société polythéiste reconnaît l'existence d'une pluralité de dieux (et même de dieux auxquels on ne croit pas) sans qu'il y ait contradiction entre ces dieux, la reconnaissance polythéiste du dieu X n'est pas contradictoire avec la reconnaissance du dieu Y, ce qui n'est évidemment pas le cas lorsque l'on affirme l'existence d'un Dieu unique ${ }^{27}$. Une telle lecture fait donc de la piété sceptique un cas particulier d'action impliquant une croyance compatible avec le scepticisme.

Le contexte de $P H$ I, 23-24, cependant, ne permet pas de donner un statut particulier à la question de la piété. Que la position de Sextus puisse être remise en cause comme incohérente d'une manière générale au nom d'une philosophie de l'action qui montrerait la nécessité d'avoir une croyance pour agir, c'est un autre problème ; ce qui importe ici, c'est la

27 Annas 2011, voir aussi la discussion dans Thorsrud 2011. 
réduction sceptique de la religion à la piété, c'est-à-dire à un ensemble d'actes pieux sans rapport avec la croyance ou l'incroyance. Le sceptique accomplit les gestes de la piété de la société dans laquelle il vit parce qu'il vit comme tout le monde, en suivant les phénomènes et la vie quotidienne. S'il se singularise, ce n'est pas par son comportement, du moins pas par son comportement extérieur. Il faut donc, comme le dit $A M$ IX, 49, servir et respecter les dieux suivant les coutumes traditionnelles, c'est-à-dire suivre les gestes et les rites de la religion populaire, de la religion civique, ou de la religion de la communauté à laquelle on appartient. On ne peut donc attendre ni prescription, ni description des rites de la piété, puisque cette solution suppose d'accepter en droit tous les rites, tous les gestes, pourvu qu'ils entrent dans le cadre des «lois et des coutumes ». La piété sceptique est donc une variable conditionnée par l'état de la société et de la culture dans lequel le sceptique vit.

\section{Les mots de la piété}

Sextus donne cependant des exemples qui dépassent le strict cadre de l'action. Ces exemples ne décrivent pas seulement des attitudes mais impliquent des énoncés typiquement dogmatiques, comme «les dieux existent» (PH I, 23-24 ; III, 2 ; AM IX, 49) et même «ils exercent une providence » (AM IX, 49 ; III, 2). N'y a-t-il pas là un retour du dogmatisme ou de la croyance, puisqu'il ne s'agit pas au sens strict d'un comportement mais d'un discours, et même d'une affirmation ? Contrairement aux «expressions sceptiques », ces affirmations ne peuvent pas être réduites à des apparences relatives : Sextus ne dit pas «les dieux paraissent exister» ou «il semble que les dieux exercent une providence», mais bien «les dieux existent $»^{28}$.

Ces exemples constituent donc un cas particulier dans le lexique sceptique, puisqu'ils mobilisent des énoncés typiquement dogmatiques (parce qu'ils portent sur des objets obscurs, et parce qu'ils ont la forme d'énoncés purement dogmatiques). Pourtant Sextus ne voit aucune contradiction entre ces énoncés et sa propre position. Et, en effet, la contradiction peut être évitée à condition d'avoir une compréhension pragmatique de ces énoncés : si nous les envisageons uniquement sous l'angle de l'attitude et des dispositions qu'ils impliquent et non comme l'expression d'une opinion ${ }^{29}$.

De quelle attitude s'agit-il ? Deux compréhensions sont possibles : on peut considérer qu'il ne s'agit que d'une attitude extérieure : le sceptique se contenterait de prononcer les mêmes mots que les croyants de manière purement extérieure, en suspendant en son for intérieur son jugement sur l'objet de la croyance. Cette interprétation limite l'acte de langage à une pure répétition formelle des mots du croyant, comme un perroquet bien dressé ${ }^{30}$. Cette interprétation n'est pas si éloignée, dans le contexte de la croyance religieuse, de ce que j'ai appelé l'interprétation libertine. Il s'agirait simplement de donner des gages à une pression sociale en répétant avec la majorité une leçon bien apprise. Mais une autre interprétation reste possible : on peut considérer que cette attitude extérieure dépasse les mots proférés, et qu'elle

${ }^{28}$ Sur la relativisation des expressions sceptiques, cf. PH I, 207. On pourrait objecter que Sextus veut dire « il me semble que les dieux existent»; mais d'une part précisément Sextus ne le dit pas - il dit « en suivant sans soutenir d'opinions les règles de la vie quotidienne, nous disons qu'il existe des dieux, nous révérons les dieux, et nous affirmons qu'ils exercent une providence » $(P H \mathrm{I}, 3)$, d'autre part son argument s'appuie non pas sur l'expérience subjective de l'existence d'un dieu ou d'une providence, mais sur l'observation selon laquelle les hommes vivent et agissent en suivant certains usages et certaines traditions, notamment religieuses. Ce qui m'apparaît phénoménalement, ce n'est donc pas l'existence des dieux ou d'une providence, mais le fait que les hommes croient à l'existence des dieux ou d'une providence.

${ }^{29}$ Pour une comparaison entre Sextus et Austin sur les actes de langage, cf. Corti 2009, chap. 2.

${ }^{30}$ Sur cette compréhension, cf. Glidden 1994. 
inclut un ensemble de gestes, d'attitudes et même une éthique. Affirmer que le sceptique dit que les dieux existent ou qu'il y a une providence, signifierait que le sceptique agit de la même façon que l'homme pieux, en respectant les mêmes normes morales et religieuses que celles du croyant. Là encore ce modèle peut s'appuyer sur la reconnaissance sceptique de la valeur de l'expérience dans laquelle s'est sédimentée la seule chose qui vaille d'un point de vue sceptique : des attitudes, des dispositions, des actions légitimées par le temps passé et leur transmission séculaire plus que par des raisons qui prétendraient les fonder.

Le choix entre ces deux interprétations comporte une part d'indécidable : s'agissant de la conviction intérieure, on peut dire ce que disait Gassendi à propos d'Épicure : « seul celui qui scrute les cœurs et les reins peut savoir et le dire ${ }^{31}$. Et il est vrai que Sextus donne peu d'éléments pour penser cette attitude. Néanmoins, si on regarde les exemples donnés par Sextus, et notamment la présence de l'affirmation de l'existence de la providence dans ces actes de langage, il semblerait que Sextus ait à l'esprit une attitude qui ne se résume pas à une simple répétition formelle et extérieure d'un credo. En effet, l'affirmation de l'existence de la providence renvoie à un véritable comportement cohérent avec l'existence d'une providence. Sextus Empiricus pourrait reprendre ici d'ailleurs un lieu commun de l'anti-épicurisme sur la négation épicurienne de la providence ${ }^{32}$ : la croyance dans la providence n'implique pas simplement la physique, mais aussi l'éthique. L'homme qui croit dans la providence agit avec confiance et respect vis-à-vis du monde et de la Cité, il ne saurait céder au désespoir. Pour un sceptique, dire qu'il y a une providence, c'est renvoyer au consensus social selon lequel ce type d'attitude est meilleur qu'une autre, c'est renvoyer à un ensemble d'actions comme s'il y avait une providence $e^{33}$.

Là encore la comparaison avec la philosophie épicurienne s'impose : là où les épicuriens adoptent des positions philosophiques dogmatiques (affirmation de l'existence des dieux ; refus de la providence), les sceptiques suspendent leur jugement; là où les épicuriens proposent une réforme de l'attitude religieuse (critique de la piété populaire, critique de la croyance en la providence), les sceptiques affirment suivre les lois et les coutumes. Les phénomènes, la vie quotidienne et notre rapport empirique à elle dictent au sceptique un ensemble de comportements et lui permettent d'agir comme tout le monde, en reprenant des attitudes qui reposent sur des convictions dogmatiques sans en reprendre les croyances.

\section{Quel type de croyant est le sceptique ?}

Une dernière objection peut être faite à Sextus : la piété ne suppose-t-elle pas une certaine force de conviction ? Si la Bible met en garde contre une forme d'adhésion tempérée (Dieu « vomit les tièdes $»^{34}$ ), c'est parce qu'on peut douter qu'elle suffise à produire l'action pieuse. Dans quelle mesure peut-on prétendre avoir une action efficace sans détermination, sans croyances et opinion fortes qui justifient un engagement ${ }^{35}$ ?

${ }^{31}$ Gassendi 2006, IV, 4, 2, t. II, p. 23.

32 Cf. Fgt Us. 368, notamment Plotin, Ennéades II, 9, 15 où la négation épicurienne de la providence laisse place à la satisfaction égoïste du plaisir. Sur l'accusation d'athéisme portée contre les Épicuriens, cf. Spinelli 2016, à paraître.

${ }^{33}$ Cf. e.g. Marc-Aurèle, Pensées II, 11.

34 Penelhum 1983, 293 qui cite l'Apocalypse de Jean III, 15 : « Je sais quelles sont vos œuvres ; que vous n'êtes ni froid ni chaud. Que n'êtes vous ou froid ou chaud!» (trad. de Sacy).

35 Il me semble que c'est la question que pose Pascal dans le fgt 525 (Lafuma) des Pensées : «Montaigne a tort. La coutume ne doit être suivie que parce qu'elle est coutume, et non parce qu'elle soit raisonnable ou juste, mais le peuple la suit par cette seule raison qu'il la croit juste. Sinon il ne la suivrait plus quoiqu'elle fût coutume, car on ne veut être assujetti qu'à la raison ou à la justice. La coutume sans cela passerait pour tyrannie, 
Dans ce contexte, la question de la nature des croyances religieuses envisagées par Sextus est intéressante. En effet, cette objection est liée à une représentation de la religion comme impliquant des croyances fortes, un credo clairement énoncé, non négociable qui serait au fondement de la plupart de nos actions. En contexte religieux, il est probable que l'adhésion décrite par Sextus ne soit pas suffisante, précisément parce qu'elle désinvestit le champ de la conviction et de l'obéissance aux dogmes pour investir celui d'une obéissance d'un autre ordre, l'obéissance aux phénomènes et à la vie quotidienne ${ }^{36}$. L'action pieuse dogmatique serait réservée au croyant déterminé, c'est-à-dire à l'homme qui déterminera son action en fonction de son adhésion à une série de dogmes.

C'est à ce niveau précisément que se situe la limite de la piété sceptique, et la critique sceptique de la religion. Si être religieux c'est avoir ce type d'engagement, alors l'homme religieux est précisément un dogmatique qui cherche à vivre en accord avec un ensemble de thèses, à en faire un guide pour la vie, déterminant des valeurs, et partant une conduite d'action disponible pour toute situation. Le fait que la piété sceptique soit une pragmatique donne la mesure de l'engagement sceptique dans les actions pieuses : son engagement sera lié à la part de nécessité portée par les lois et les coutumes; et cette nécessité n'est pas nécessairement à comprendre comme une contrainte physique à la manière de l'ordre du tyran d'AM XI, 164, mais plutôt comme une obligation sociale bien comprise ${ }^{37}$.

mais l'empire de la raison et de la justice n'est non plus tyrannique que celui de la délectation. Ce sont les principes naturels à l'homme».

${ }^{36} \mathrm{Cf}$. $P H$ I, 230 où Sextus décrit le mode de persuasion qui touche le sceptique comme « ne pas résister mais suivre simplement sans forte inclination ni penchant, comme on dit qu'un enfant est persuadé par son pédagogue ».

${ }^{37} \mathrm{Je}$ remercie Anne-Isabelle Bouton-Touboulic et Carlos Lévy pour leur invitation au colloque de Bordeaux auquel je n'ai malheureusement pas pu assister. Je remercie Sylvia Giocanti et Emidio Spinelli pour leurs remarques et leurs critiques. 
